\title{
Development of the Smart Metal Casting Process Management System
}

\author{
Jung-Sook Kim ${ }^{1}$, Jae-Hyeong Kim, Jun-Ho Jeong and Jang-Young Chung ${ }^{2}$ \\ ${ }^{1}$ Division of IT, Kimpo College, Gimpo-si, Geyonggi-do, Korea \\ ${ }^{2}$ Department of Computer Engineering, Dongguk University \\ Seoul, Korea \\ kimjs@kimpo.ac.kr
}

\begin{abstract}
In this paper, we describe the smart metal casting process management system, in which we applied case-based reasoning to find a near optimal process route on the window environment. The metal casting process is complex and variable depends on a kind of metal casting products. Especially, the metal casting industry has a feature which produces small quantities but produces a lot of different types of metal casting products. And we developed the smart metal casting process management system which could show the process route according to the product cases intelligently using the result of case-based reasoning. The experimental result shows that our metal casting process management system schemes achieves more productivity than manual management schemes.
\end{abstract}

Keywords: Metal Casting, Smart Process Management System, Case-Based Reasoning, Manufacturing Process Management

\section{Introduction}

Manufacturing Process Management (MPM) represents a huge step in the quest to link product design with production electronically, in order to improve information quality and reduce time-to-market. The companies surveyed that do rely on computers use Microsoft Excel spreadsheets stored on local computers, lacking integration to other systems. Most of these spreadsheets are fairly sophisticated, involve hundreds of hours of development time by process engineers, and require considerable regular maintenance. The most common workflow for passing product information to production planning relied on paper forms of independent spreadsheets with the information manually entered into the production-planning (MRP/ERP/MES) systems by low-wage clerks. This manual process has led to process-time delays as well as to inconsistencies in the detailed information, especially as it relates to model-mix and optional-work content. The downstream implication of these information delays and inaccuracies is that the production planning systems cannot accurately plan and manage production. Thus, the playback from the significant investment in these systems cannot be realized. Computerized MPM systems can solve these problems. MPM systems can take the engineering bill of materials (eBOM) from product engineering electronically, and provide the information to industrial and manufacturing engineers, who can design and document the manufacturing processes at high level of detail, while mapping the processes to manufactured and consumed components. In addition, the important attributes of production sequence and precedence are documented; costs are calculated for manufactured activity, and value-added work content is determined $[1,2,3]$. 
Metal casting is one of the most common casting processes. Metal patterns are more expensive but are more dimensionally stable and durable. Metallic patterns are used where repetitive production of castings is required in large quantities. Casting is a manufacturing process by which a liquid material is usually poured into a mold, which contains a hollow cavity of the desired shape, and then allowed to solidify. The solidified part is also known as a casting, which is ejected or broken out of the mold to complete the process. Casting materials are usually metals or various cold setting materials that cure after mixing two or more components together; examples are epoxy, concrete, plaster and clay. Casting is most often used for making complex shapes that would be difficult or uneconomical to make by other methods. Also, the metal casting process has a complex and various processing route. At this point, the kinds of desired shape are very various. Also, the required component properties are different. So, the casting process management system supports the user in component design, the determination of melting practice and casting methods to pattern and mold making, heat treatment, and finishing using case-based reasoning $[4,5,6]$. This saves costs along the entire casting manufacturing route. Also, this can reduces the error rate of products and a producer must increase the productivity. In this paper, we develop the smart metal process management system. The structure of this paper is as follows. Section 2 briefly introduces related studies. Section 3 describes the smart metal process management system and Section 4 presents its implementation and results details. Finally, the conclusions are drawn and the future study is discussed in Section 5.

\section{Related Works}

\section{A. Manufacturing Process Management}

MPM represents a huge step in the quest to link product design with production electronically, in order to improve information quality and reduce time-to-market. Major strides have been made in recent years with the deployment of commercial applications capable of integrating the complicated and configurable BOM (Bill of Material) with that of an equally complicated BOP (Bill of Process), within an environment that encourages the evaluation of alternatives and the development, storage, and comparison of temporarily infeasible scenarios, in search of optimal designs. The greatest benefit from MPM can be seen in improved production efficiencies derived from more tightly designed and managed production systems. It is not uncommon to see assembly line efficiencies increase by ten percent in mixed-model environments. New or modified product launch times have been reduced by 25 to $50 \%$ primarily due to the availability of instant information, and to the benefits of editing existing, similar process, rather than re-engineering from scratch as is often done. More importantly, MPM applications provide the analytical and data management abilities necessary for companies to move to mixed-model production system in an effort to reduce Work-in-Progress (WIP) and finished goods inventory, and to improve overall product quality and production responsiveness.

Benefits can be seen with improved access, management, and accuracy of production engineering information for downstream applications such as MES, MRP, and ERP systems. By providing detailed manufacturing process information throughout the organization as well as to downstream applications, the organization now has the ability to distribute best practices quickly and accurately throughout the global organization, and to react intelligently to concurrent changes in products and processes. Three primary tasks govern the processes in a factory from product design through production. Manufactures design what they will make, they design how they will make the product, and they plan when they will make it. The what and when in manufacturing have evolved into multi-billion-dollar software and service 
industries. Few companies design their products or plan their manufacturing schedules on paper anymore. However, the same cannot be said for the design of how products are made [1].

\section{B. Case-Based Reasoning}

Case-based reasoning is an AI (Artificial Intelligence) paradigm that can be synergistically combined with other approaches to facilitate a broad array of tasks. Case-based reasoning is the process of using past cases to interpret or solve new problem cases. Central to case-based reasoning is the determination of which past cases are similar enough to the new case to warrant consideration for reuse in the new problem. In case-based reasoning terminology, a case usually denotes a problem situation. A previously experienced situation, which has been captured and learned in a way that it can be reused in the solving of future problems, is referred to as a past case, previous case, stored case, or retained case. Correspondingly, a new case or unsolved case is the description of a new problem to be solved. Case-based reasoning is, in effect, a cyclic and integrated process of solving a problem, learning from the experience, and solving a new problem, etc. There are two basic kinds of CBR: interpretive case-based reasoning and problem solving. Interpretive case-based reasoning involves the use of past cases, typically called precedents, to create an analysis and justification for the interpretation of a new case. Problem-solving case-based reasoning involves adapting a solution to a past problem, typically a design or plan, to meet the requirements of the new situation. All case-based reasoning follows the same basic steps: (1) analyze the new case (or problem); (2) based on this analysis, retrieve relevant past cases from a case base; (3) based on a 'similarity metric,' rank retrieved cases according to how relevant or useful they are with respect to the new case, and select one or more of the best cases to use in solving the new case; (4) create a solution for the new case; (5) test and explore the proposed solution; and (6) if appropriate, add the new case and its solution to the case base and index it so that it can be retrieved for future use [6].

\section{Smart Metal Casting Process Management System}

\section{A. Smart System}

In order to apply case-based reasoning, we need past cases, and we tried to collect the cases for such instances but we couldn't find them. And, we selected the representative past cases first hand. We expressed the collected cases, and stored them in a case base. After that, we retrieved relevant past cases from the case base, using a metal casting product ID in searching for the optimal process management of the metal casting industry.

\section{B. DataBase Schema}

The MPM data model is process-centric, with strong and bi-directional relations for producing and consuming components to the product's eBOM. Along with the product and process data repositories, there are plant and resource data stores that form the basis of the MPM data model. With four primary data stores, the method of reconciliation needs to be flexible and variable by customer, or by installation. To save a lot of materials or event information, we developed a database for the metal casting process management system. The following, Figure 1, shows a database schema which has eight tables. The Figure 1 shows the detailed ordering information table fields, such as a serial number, an ordering number, a product number and a lot number, etc. 


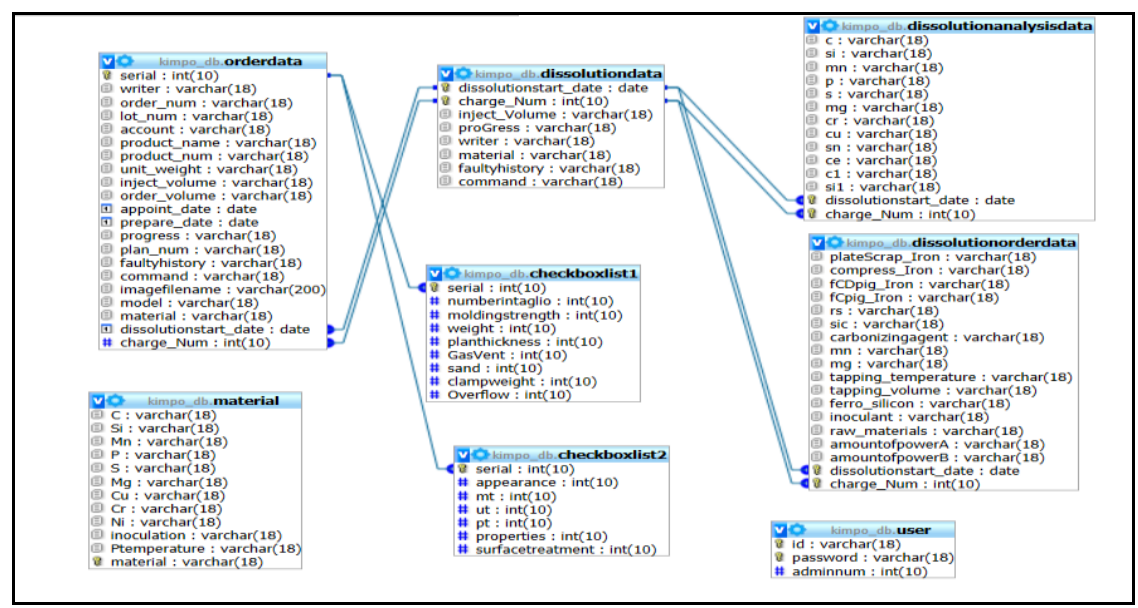

Figure 1. Database schema

\section{Implementation and Results}

We have implemented a smart casting process management system with C\# based on Windows. The window dialog control function can provide an easy and convenient method of operating the metal casting processing management system because the operator has much user experience with window dialog, through other window dialog. Figure 2 shows the order information to make the metal casting products. The operator inputs the information in the field such as an ID of product, a kind of materials, requirements of customer, a drawing of product, and etc.

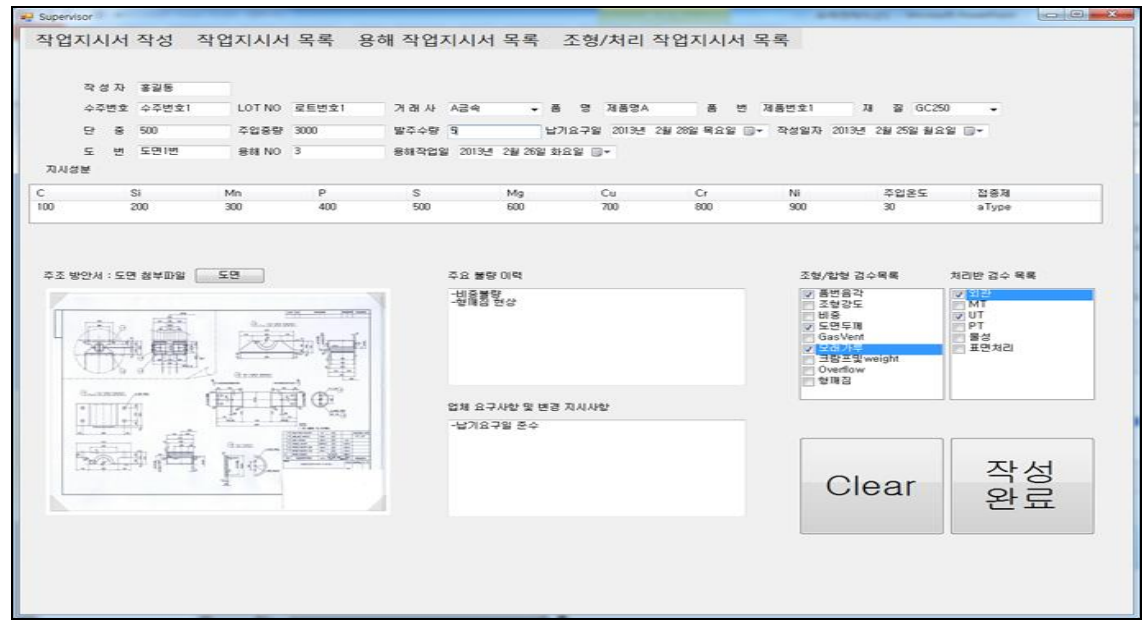

Figure 2. Order window dialog

MPM applications drive mixed-model applications by using the configured product BOM with optional content and filtering it though a model-mix scenario. The appropriate tasks can then be assigned by location, and resources can be allocated in appropriate quantities. This engineering effort involves the simultaneous optimization of: part and process precedence; location sequence in the case of assembly lines; resource availability in terms of the right person and machine at the right place; zone requirements in terms of cluster work, e.g., inside, outside, or underneath the product. The effort seeks to minimize throughput time, non-value- 
added work content, and resource requirements. New MPM-based mixed-model line balancing applications can largely solve these problems automatically, and thus require a minimum amount of manual interaction to produce highly efficient and entirely accurate solutions [1]. The following Figure 3 shows the process precedence, location sequence of assembly line, and resource availability.

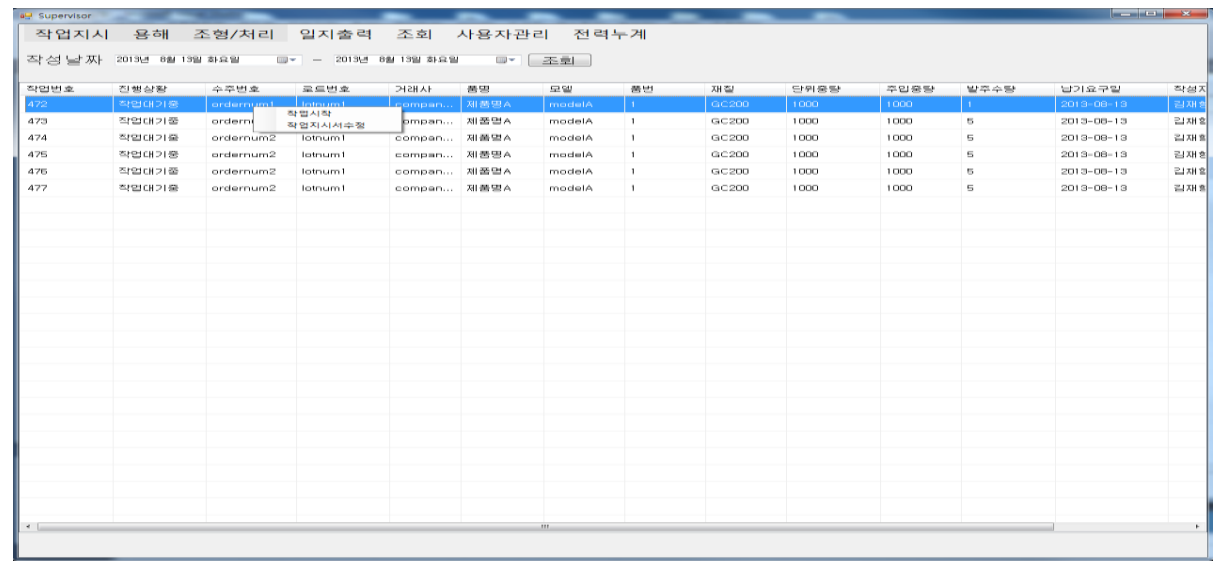

Figure 3. Process precedence list

One major benefit of the BOM in line balancing derives from the ability to use the product structure to define the default process precedence, and detailed activity-based work content for each model and option -accurately, automatically, and effortlessly [1]. As Figure 2 shows, the smart metal casting process management system presents the optimal manufacturing route depends on a kind of the products based on the result of case-based reasoning. The red color buttons represent the processing steps of the given casting product route, but the grey color buttons represent the skipping steps.

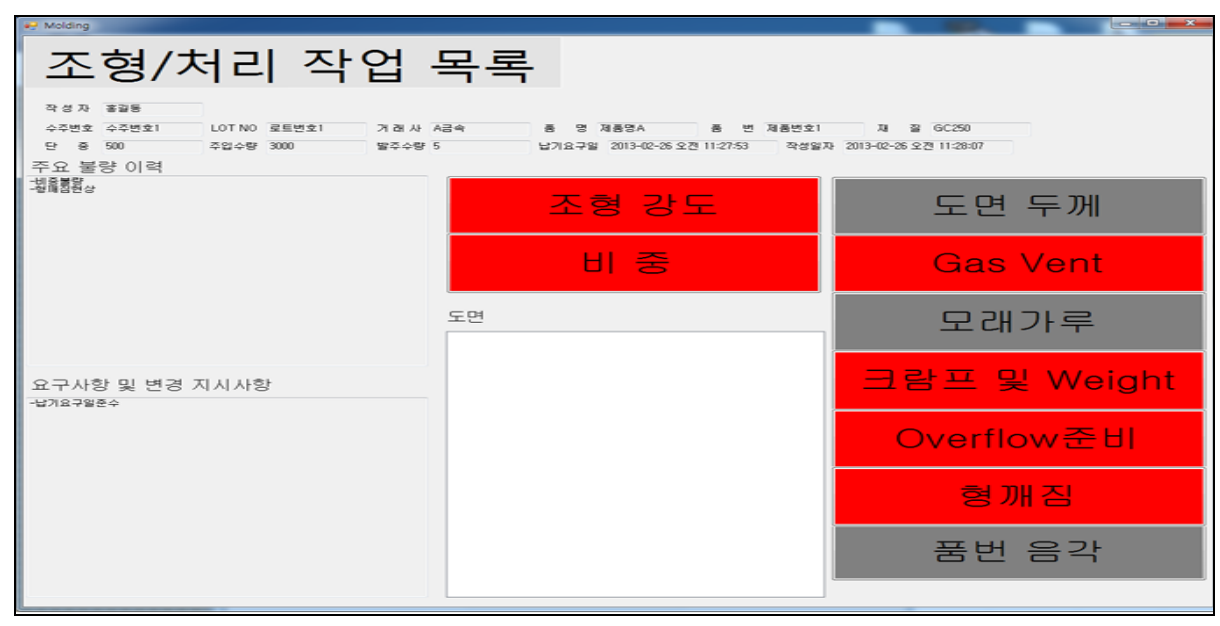

Figure 4. Finishing process dialog

You can see the result of dissolution process in Figure 4. After the finishing process, the button color change red into green in order to notify the finishing process done. 


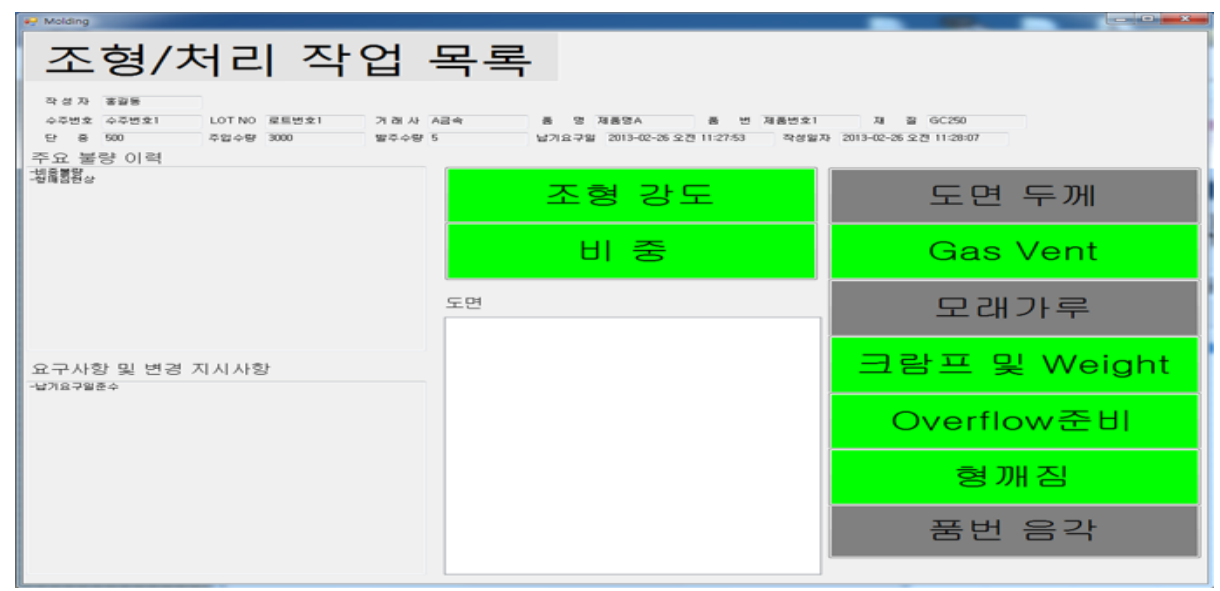

Figure 5. Result of finishing process

Prior methods relying on word-of-mouth or three-ring binders are not sufficient to ensure that operators have accurate information when they need it. As such, it is becoming more popular to establish electronic shop floor information systems that often include terminals or touch-screen displays at many (if not all) workstations on the shop floor. The technology involved with installing these systems is minor compared to the effort required to develop, distribute, and maintain the information requested by these systems [1]. The following Figure 6 shows the report form for accurate information of process management.

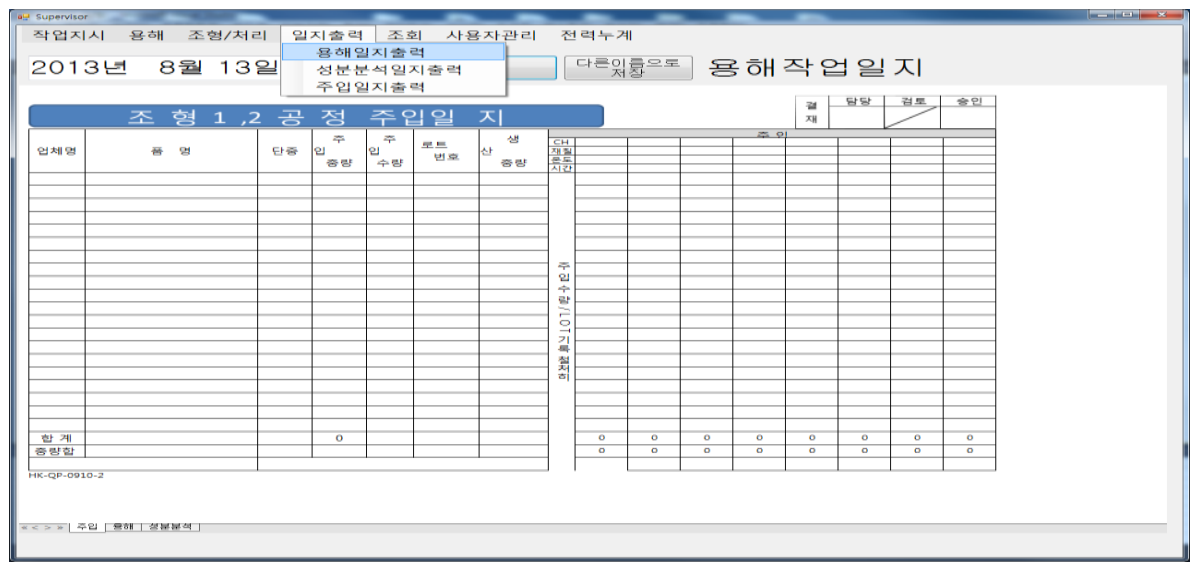

Figure 6. Report form

\section{Conclusions and Future Work}

MPM systems can take the engineering bill of materials from product engineering electronically, and provide the information to industrial and manufacturing engineers, who can design and document the manufacturing processes at high level of detail, while mapping the processes to manufactured and consumed components. In addition, the important attributes of production sequence and precedence are documented; costs are calculated for manufactured activity, and value-added work content is determined. In this paper, we have implemented a smart metal casting process management system using case-based reasoning with $\mathrm{C} \#$ based on Windows. The window dialog control function can provide an easy and 
convenient method of operating the metal casting process management system because the operator has much user experience with window dialog, through other window dialog. The experimental result shows that our metal casting process management system schemes achieves more productivity than manual management schemes. In the future study, we are going to extend this research to apply broadly to traditional metal casting industry in order to connect the sensors, such as a temperature and an angle, based on wireless communication.

\title{
Acknowledgements
}

This work (Grants No. C0028922) was supported by Business for Cooperative R\&D between Industry, Academy, and Research Institute funded Korea Small and Medium Business Administration in 2012.

\section{References}

[1] D. Sly, "Manufacturing Process Management(MPM)", Technology Trends in PLM by CPDA, (2004) June, pp. 1-8.

[2] K. Kerdprasop and N. Kerdprasop, "Performance Analysis of Complex Manufacturing Process with Sequence Data Mining Technique", International Journal of Control and Automation, vol. 6, no. 3, (2013), pp. 301-312.

[3] H. Faris, "A Symbolic Regression Approach for Modeling the Temperature of Metal Cutting Tool", International Journal of Control and Automation, vol. 6, no. 4, (2013), pp. 431-440.

[4] http:// http://en.wikipedia.org/wiki/Casting, (2013).

[5] E. P. Degarmo, J. T. Black and R. A. Kohser, "Materials and Processes in Manufacturing (9th ed.)", Wiley, (2003).

[6] A. Aamodt and E. Plaza, "Case-based Reasoning: Foundational Issues, Methodological Variations, and System Approaches”, IOS Press, Artificial Intelligence Communications, vol. 7, no. 1, (1994), pp. 39-59.

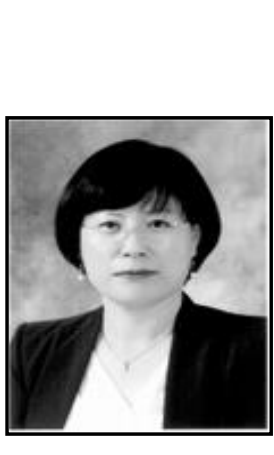

\begin{abstract}
Authors

\section{Jung-Sook Kim}

Jung-Sook Kim received the B.S., M.S., and Ph.D. degrees in computer engineering from Dongguk University, Seoul, Korea in 1993, 1995 and 1999, respectively. She is a professor in department of multimedia at Kimpo College. Her research interests include in the fields of intelligent systems, IT convergence, and distributed and parallel system.
\end{abstract}

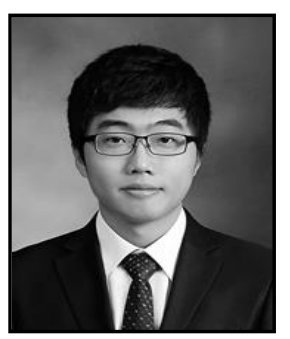

\section{Jae Hyeong Kim}

Jae Hyeong Kim received a B.S. degree in computer engineering from Dongguk University, Seoul, Korea in 2013. He is currently a master's degree in computer engineering at Dongguk University. His research interests include in authentication protocol, data privacy, parallel encryption, and cloud security. 


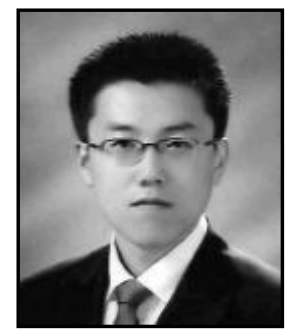

\section{Junho Jung}

Junho Jeong received the B.S. and M.S. degree in computer engineering from Dongguk University, Seoul, Korea in 2007 and 2009 respectively. $\mathrm{He}$ is a doctoral candidate in computer engineering at Dongguk University. His research interests include in information security system, distributed processing system, distributed and parallel algorithms, and cloud security.

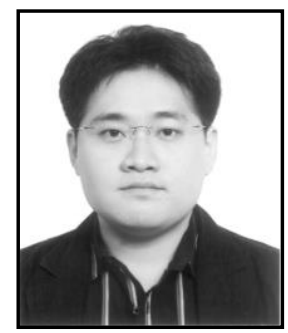

\section{Jang-young Chung}

Jang-young Chung received a B.S. degree in Computer \& Information Security from Deajeon University, Deajeon, Korea in 2006, and received a M.S. degree in computer engineering from Dongguk University, Seoul, Korea in 2009. He is a doctoral candidate in computer engineering at Dongguk University. His research interests include image security, authentication protocol, data privacy, parallel encryption, cloud security, and biometric security. 